\title{
Apoptosis in Skeletal Myocytes: A Potential Target for Interventions against Sarcopenia and Physical Frailty - A Mini-Review
}

\author{
Emanuele Marzetti ${ }^{a}$ Riccardo Calvani ${ }^{b}$ Roberto Bernabei ${ }^{b}$ \\ Christiaan Leeuwenburgh ${ }^{c}$ \\ Departments of a Orthopaedics and Traumatology, and ${ }^{\mathrm{b}}$ Gerontology, Geriatrics and Physiatrics, University \\ Hospital Agostino Gemelli, Catholic University of the Sacred Heart, Rome, Italy; ${ }^{\circ}$ Department of Aging and \\ Geriatric Research, Institute on Aging, University of Florida, Gainesville, Fla., USA
}

\section{Key Words}

Muscle $\cdot$ Mitochondria $\cdot$ Calorie restriction $\cdot$ Exercise $\cdot$

Resveratrol $\cdot$ Angiotensin-converting enzyme inhibitors ·

Hormones

\begin{abstract}
Background: Sarcopenia, the age-related loss of muscle mass and function, represents a relevant public health issue due to its high prevalence and detrimental consequences. While the exact mechanisms underlying the pathogenesis of sarcopenia are not clear, growing experimental evidence indicates that progressive myonuclear elimination over the course of aging via an apoptosis-like process may represent a converging mechanism through which muscle atrophy and loss of physical function develop. Notably, the proapoptotic environment taking place in aged muscle appears amenable to interventions. Objective: We aimed at providing (1) an overview of signaling pathways of apoptosis relevant to sarcopenia, and (2) a review of the literature supporting myocyte apoptosis as a target for interventions against muscle aging. Methods: We summarized findings from studies focused on skeletal myocyte apoptosis as a mechanism in the development of sarcopenia and reports supporting
\end{abstract}

myonuclear apoptosis as a target for interventions against age-related muscle loss. Results: Advanced age is associated with increased signaling through extrinsic and intrinsic apoptotic pathways in skeletal myocytes. In contrast, downregulation of myocyte apoptosis through calorie restriction, exercise training, hormonal supplementation, drugs (e.g. angiotensin-converting enzyme inhibitors, acetaminophen, antimyostatin antibodies), nutraceuticals or genetic interventions (e.g. PGC- $1 \alpha$ overexpression) is linked with preservation of muscle integrity and improved physical performance in late life. Preliminary data also indicate that skeletal myocyte apoptotic signaling may be downregulated by compounds, such as resveratrol, with calorie restrictionmimicking properties. Whether exercise mimetics exert a similar effect has not yet been investigated. Conclusions: Available evidence suggests that targeting myonuclear apoptosis might provide novel and effective therapeutic tools to combat sarcopenia. Further research is required to definitely establish if downregulating myonuclear apoptosis is effective in maintaining muscle mass and function in late life, identify the most relevant apoptotic pathway(s) to target, and determine the optimal timing for intervening.

Copyright $\odot 2011$ S. Karger AG, Basel

Emanuele Marzetti, MD, PhD

Department of Orthopaedics and Traumatology, University Hospital Agostino Gemelli Catholic University of the Sacred Heart, Largo A. Gemelli 1

IT-00168 Rome (Italy)

Tel. +39063015 5669, E-Mail emarzetti@live.com 


\section{Introduction}

Sarcopenia is a geriatric syndrome encompassing the loss of muscle mass and strength or physical performance with age [1]. Relevant consequences of sarcopenia include impaired mobility, higher incidence of falls and fractures, reduced quality of life, greater social and healthcare needs, and increased morbidity and mortality. Furthermore, sarcopenia is a major determinant of frailty [2], i.e. a condition of impaired homeostatic reserve and reduced capacity of the organism to withstand stress, resulting in increased vulnerability to adverse health outcomes.

Given the clinical and socioeconomic ramifications of sarcopenia, extensive research has been devoted to understanding the biological determinants of this syndrome in order to provide specific targets for therapeutic and/or preventive interventions. In this context, experimental evidence supporting a role for myocyte apoptosis in the pathogenesis of sarcopenia has accumulated over the last decade [3]. Specifically, it is hypothesized that the acceleration of apoptosis taking place in the aging muscle may represent a converging mechanism through which muscle atrophy and physical function decline ensue. Indeed, the extent of apoptosis increases in the skeletal muscle over the course of aging, paralleling the loss of muscle mass and strength [3]. Furthermore, the biochemical characterization of experimental models of acute muscle atrophy has revealed elevated levels of myocyte apoptosis [4]. In addition, the genetic characterization of IL-10-deficient mice, a rodent model of frailty, unveiled upregulation of several apoptosis-related genes in the skeletal muscle [5], further supporting the involvement of accelerated myocyte apoptosis in the pathogenesis of sarcopenia and physical function loss in late life. Conversely, downregulation of skeletal myocyte apoptotic signaling via pharmacological, behavioral or genetic interventions has been associated with attenuation of muscle loss and declining physical function [3]. The molecular mechanisms responsible for the acceleration of myonuclear apoptosis at old age have not yet been clearly established. However, it is believed that oxidative stress [6], chronic inflammation [7], reduced anabolic drive [8] and impaired insulin sensitivity [9] may play a prominent role.

In the next sections, an overview of signaling pathways of apoptosis relevant to sarcopenia will be provided, followed by a brief review of the literature supporting myocyte apoptosis as a target for interventions against muscle aging.

\section{Signaling Pathways of Apoptosis and Peculiarities of Apoptosis in Skeletal Myocytes}

Apoptosis is a process of programmed cell death which proceeds through a highly coordinated set of events, resulting in cellular self-destruction without inflammation or damage to the surrounding tissue [10]. For a detailed description of apoptotic signaling pathways, the reader is referred to comprehensive specialized reviews [11, 12]. Broadly, the apoptotic machinery comprises regulatory proteins, endonucleases, protease inhibitors and proteolytic enzymes, known as caspases. Upon cell death stimulus, initiator caspases (i.e. caspase- $8,-9$ and -12) are engaged, leading to the activation of effector caspases (i.e. caspase- $3,-6$ and -7), which are responsible for cellular degradation and DNA fragmentation. Two major pathways of caspase activation are distinguished based on the extrinsic or intrinsic origin of the death-inducing stimulus (fig. 1). The extrinsic pathway is triggered by the interaction of cell surface death receptors (e.g. tumor necrosis factor receptor, TNF-R) with their ligands (e.g. TNF- $\alpha$ ). The intrinsic pathway involves the participation of mitochondria or the endoplasmic reticulum. Notably, mitochondria can induce apoptosis independent of caspase activation through the release of apoptosis-inducing factor and endonuclease $\mathrm{G}$, both of which can directly operate DNA fragmentation.

In the skeletal muscle, given the multinucleated nature of myocytes, apoptosis displays unique features. Indeed, the activation of the apoptotic cascade results in the removal of individual myonuclei and the relative portion of sarcoplasm, a process known as myonuclear apoptosis. This pathway leads to fiber atrophy rather than wholesale cell death. In addition, apoptotic signaling may stimulate muscle protein degradation through the activation of the ubiquitin-proteasome system, leading to fiber atrophy, independent of myonuclear removal [13]. Indeed, muscle proteolysis and apoptosis are intimately interconnected given that apoptotic signaling is required for and precedes protein degradation during muscle atrophy [14]. Another peculiarity of skeletal myofibers resides in the presence of two bioenergetically and structurally distinct mitochondrial subpopulations: subsarcolemmal mitochondria, located beneath the sarcolemma, and intermyofibrillar mitochondria, arranged in parallel rows between the myofibrils. These two subpopulations display different susceptibility towards apoptotic stimuli and may therefore be differentially involved in the pathogenesis of sarcopenia [4]. 


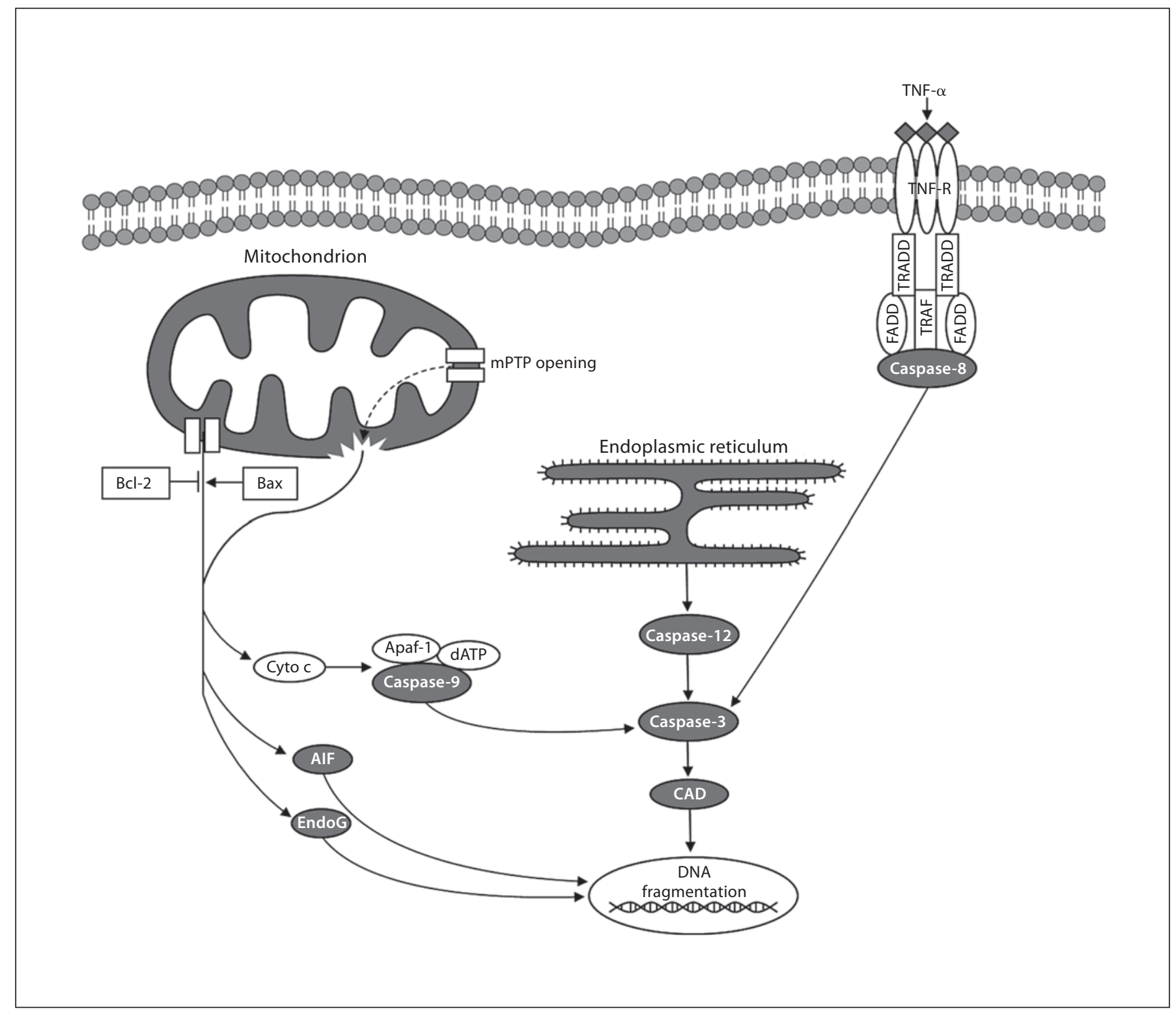

Fig. 1. Schematic overview of major extrinsic and intrinsic apoptotic signaling pathways. Binding of TNF- $\alpha$ to its cell membrane receptor (TNF-R) initiates the extrinsic apoptotic pathway through the recruitment of adaptor proteins (e.g. FADD, TRADD, TRAF). The resulting death-inducing signaling complex engages caspase-8, which subsequently activates caspase- 3 . The mitochondrial intrinsic apoptotic pathway involves the release of apoptogenic factors from the intermembrane compartment. This process is regulated by the balance between pro- and antiapoptotic Bcl-2 family proteins, such as Bax and Bcl-2, respectively. In addition, opening of the mitochondrial permeability transition pore (mPTP) can induce a sudden increase in membrane permeability, collapse of membrane potential, mitochondrial swelling and rupture of the outer membrane, with subsequent release of death effectors. For instance, following outer membrane permeabilization, cytochrome $c$ can complex with Apaf-1, dATP and caspase-9, forming an apoptosome, which engages caspase-3. Alternatively, mitochondria can release AIF and EndoG, which perform DNA fragmentation independent of caspase activation. Finally, the endoplasmic reticulum-mediated intrinsic apoptotic pathway is initiated by the engagement of caspase-12, followed by caspase-3 activation. AIF = Apoptosis-inducing factor; Apaf-1 = apoptotic protease-activating factor-1; Bcl-2 = B-cell lymphoma-2; $\mathrm{Bax}=\mathrm{Bcl}-2$-associated $\mathrm{X}$ protein $\mathrm{CAD}=$ caspaseactivated DNase; Cyto $c=$ cytochrome $c$; EndoG $=$ endonuclease G; FADD = Fas-associated death domain; TRADD = TNF-receptor-associated death domain; TRAF $=$ TNF-receptor-associated factor. 
Table 1. Synoptic view of changes in myonuclear apoptotic signaling elicited by behavioral, pharmacological and genetic interventions

\begin{tabular}{|c|c|c|c|c|}
\hline & \multicolumn{4}{|c|}{ Apoptotic signaling pathways } \\
\hline & extrinsic & $\begin{array}{l}\text { mitochondrial } \\
\text { caspase-dependent }\end{array}$ & $\begin{array}{l}\text { mitochondrial } \\
\text { caspase-independent }\end{array}$ & $\begin{array}{l}\text { sarcoplasmic } \\
\text { reticulum pathway }\end{array}$ \\
\hline \multicolumn{5}{|l|}{ Behavioral interventions } \\
\hline $\mathrm{CR}$ & $\downarrow$ & $\downarrow$ & $\downarrow$ & $\downarrow$ \\
\hline Resveratrol (CR mimetic) & - & $\downarrow$ & - & - \\
\hline Exercise training & $\downarrow$ & $\downarrow$ & - & - \\
\hline \multicolumn{5}{|l|}{ Drugs and hormones } \\
\hline Enalapril & - & $\downarrow$ & $\leftrightarrow$ & - \\
\hline Acetaminophen & - & $\downarrow^{\mathrm{a}}$ & $\downarrow^{\mathrm{a}}$ & - \\
\hline Antimyostatin antibody & $\downarrow^{\mathrm{b}}$ & $\downarrow^{\mathrm{b}}$ & - & $\downarrow^{\mathrm{b}}$ \\
\hline $\mathrm{Q} 10$ + creatine + ginseng & - & $\downarrow^{\mathrm{a}}$ & $\downarrow^{\mathrm{a}}$ & - \\
\hline Testosterone $\mathrm{e}^{\mathrm{c}}$ & $?$ & $?$ & $?$ & $?$ \\
\hline \multicolumn{5}{|l|}{ Genetic manipulations } \\
\hline PGC-1 $\alpha$ overexpression & - & $\downarrow$ & - & - \\
\hline
\end{tabular}

\footnotetext{
$\downarrow=$ Decrease; $\leftrightarrow$ = unchanged; $-=$ not investigated.

${ }^{a}$ Apoptogenic mediators downstream of mitochondrial outer membrane permeabilization were not investigated. ${ }^{\mathrm{b}}$ Caspase-3, downstream of the extrinsic pathway as well as the mitochondrial caspase-dependent and sarcoplasmic reticulum pathways, was the only apoptogenic mediator investigated. ${ }^{c}$ Only the extent of apoptosis was determined.
}

\section{Myonuclear Apoptosis as a Target for Interventions against Sarcopenia}

Several preclinical studies suggest that myonuclear apoptosis may provide a selective biological target for the development of preventive and therapeutic interventions against sarcopenia. Indeed, it has clearly been shown that preservation or accretion of muscle mass and strength in aged rodents, elicited by behavioral, pharmacological or genetic interventions, is accompanied, and perhaps mediated, by decreases in the extent of myocyte apoptosis.

In the next sections, the effects of these antisarcopenic interventions on apoptotic signaling will be briefly discussed.

\section{Calorie Restriction and Calorie Restriction Mimetics}

Calorie restriction (CR) without malnutrition is a robust antiaging intervention that has been shown to delay the onset and attenuate the severity of sarcopenia in a variety of species [15]. Studies in old laboratory rodents have demonstrated that $\mathrm{CR}$ reduces the activation of extrinsic and intrinsic apoptotic pathways (table 1). Indeed, lifelong moderate CR (i.e. $40 \%$ reduction in calorie intake) decreased the expression of TNF- $\alpha$, TNF-R1 and Fas-as- sociated protein with death domain in muscles of old rats relative to age-matched controls fed ad libitum $[7,8]$. Furthermore, CR prevented the age-related elevation of active caspase- 8 levels, downstream of TNF- $\alpha[7,8]$. As a result, cleavage of caspase-3 as well as the extent of apoptotic DNA fragmentation were reduced in old CR rodents. Interestingly, CR-mediated downregulation of the death receptor apoptotic signaling was accompanied by higher muscular levels of IL-15 and its specific receptor subunit IL-15 receptor alpha chain (IL-15R $\alpha$ ) [8]. This muscle-derived anabolic cytokine possesses antiapoptotic properties, likely resulting from its interference with the apoptotic signaling pathway triggered by TNF- $\alpha$ [8]. Therefore, it is possible that the inhibition of death receptor-mediated apoptosis by CR may be achieved, at least partly, through the preservation of IL-15 signaling into late life.

With regard to the intrinsic apoptotic pathway, lifelong $40 \%$ CR was shown to increase the expression of apoptosis repressor with a caspase recruitment domain (ARC) in the gastrocnemius muscle of old rodents [16]. Notably, ARC, besides inhibiting caspase- 2 and -8 activity, also prevents mitochondrial outer membrane permeabilization [17]. As a result, apoptosis-inducing factor 
levels were lower in muscles from old CR rats relative to animals fed ad libitum. In addition, the expression of apoptosis protease activating factor-1, a component of the apoptosome, was reduced by CR, although this effect did not translate into decreased levels of active caspase-9. Finally, Dirks and Leeuwenburgh [16] showed that lifelong $40 \%$ CR reduced the expression of procaspase- 12 in the gastrocnemius muscle of aged rats, indicating that $\mathrm{CR}$ can also counteract sarcoplasmic reticulum stress-mediated apoptotic signaling.

Despite undisputed health benefits brought about by $\mathrm{CR}$, it is likely that most people will not be able to sustain substantial food restrictions for the long term. Moreover, weight loss may be harmful in nonobese older persons as it can accelerate muscle loss and increase the risk of disability and mortality [18]. Difficulties adhering to longterm food intake reductions and health concerns intrinsic to the adoption of CR regimens have sparked a great interest in the field of so-called CR mimetics. In fact, these agents could reproduce the effects of CR without requiring modifications in food intake.

Resveratrol, a plant-derived polyphenol found in grapes, red wine, peanuts and some berries, is probably the most promising of such compounds. Although the exact mechanisms underlying the CR-mimicking properties of resveratrol have not yet been fully unveiled, it is hypothesized that this compound may confer an increased cellular resistance to various stressors (e.g. oxidative stress and inflammation) by deflecting stress-response pathways from apoptosis toward stress resistance and survival [19]. Indeed, pretreatment with resveratrol of rat pheochromocytoma cells (PC12 cells) challenged with oxidants resulted in increased content of antiapoptotic B cell lymphoma-2 (Bcl-2), reduced expression of proapoptotic Bcl-2-associated X protein (Bax) and lower caspase-3 levels and activity [20]. Interestingly, Jackson et al. [21] found that 3-week resveratrol supplementation reduced levels of caspase- 9 and increased the expression of Bcl-2 in the gastrocnemius muscle of old rats subjected to hind limb suspension. These adaptations were accompanied by a significant attenuation of muscle force loss secondary to immobilization.

\section{Physical Exercise}

Physical exercise is currently considered the primary countermeasure to sarcopenia [22]. Both aerobic and resistance exercise training have been shown to attenuate the rate of decline in muscle mass and strength with age [22]. Moreover, resistance exercise helps even very old, frail persons retain the ability of improving muscle mass and strength as well as physical performance [23]. Adaptations elicited by physical exercise in skeletal myocytes are complex and involve protein metabolism, mitochondrial bioenergetics, autophagy and redox homeostasis. With regard to the effects of exercise training on myonuclear apoptotic signaling, Song et al. [24] showed that 12 -week treadmill exercise reduced the expression of Bax in the gastrocnemius muscle of old rats. Conversely, levels of Bcl-2 were increased in exercised rodents, resulting in a $96 \%$ decrease in the Bax-to-Bcl-2 ratio. In addition, cleavage of caspase- 3 was lowered by $95 \%$ in old exercised rats. As a consequence, the extent of gastrocnemius apoptotic DNA fragmentation was significantly attenuated by the exercise intervention, such that old trained rats displayed levels of apoptosis similar to those observed in young control animals. It is noteworthy that the reduced severity of apoptosis was accompanied by ameliorations in muscle morphology, as indicated by the increased fiber cross-sectional area and diminished extramyocyte space. Similarly, Marzetti et al. [25] found that 4-week treadmill exercise training downregulated the death receptor pathway of apoptosis in the extensor digitorum longus muscle of old rats. Indeed, the age-related increase of TNF-R1, active caspase- 8 and cleaved caspase- 3 was reversed by physical exercise, resulting in reduced levels of apoptotic DNA fragmentation. These adaptations were accompanied by improvements in exercise tolerance and forelimb grip strength.

Although exercise training is highly effective in counteracting age-related muscle loss, the large-scale implementation of such intervention is hampered by the lack of motivation of most persons. In addition, many older adults may be unable to engage in regular exercise training due to concomitant disabling conditions. To overcome such barriers, the intriguing possibility exists of pharmacologically mimicking exercise using pathwayspecific drugs. For instance, 4 -week treatment with the orally active AMP-activated protein kinase agonist AICAR enhanced running endurance in sedentary mice [26]. The possibility of synthetically mimicking physical exercise by targeting myonuclear apoptosis warrants investigation.

\section{Drugs and Hormones}

As previously discussed, although behavioral interventions, such as moderate $\mathrm{CR}$ and physical exercise, have proven beneficial against age-associated muscle loss in experimental settings, most older persons are reluctant or unable to participate in such approaches. Therefore, the identification of biological targets for pharmacologi- 
cal interventions against sarcopenia is of extraordinary relevance. In this context, we recently showed that treatment of rats with the angiotensin-converting enzyme inhibitor enalapril between 24 and 27 months of age downregulated mitochondria-mediated apoptosis in the gastrocnemius muscle [27]. Specifically, enalapril administration reduced cytosolic levels of cytochrome $c$ as well as the activation of caspase- 9 and -3 , resulting in decreased apoptotic DNA fragmentation. In contrast, the mitochondrial caspase-independent apoptotic pathway was unaffected by the intervention. Importantly, attenuation of skeletal muscle apoptosis was associated with improvements in physical performance, as determined by forelimb grip strength. Contrary to enalapril, losartan, an angiotensin receptor blocker, although producing similar effects on physical performance, did not attenuate the extent of skeletal myocyte apoptosis, suggesting that the two drugs may act through distinct cellular pathways [27].

In another recent study, administration of acetaminophen to aged rats for 6 months increased Akt/protein kinase B activity and reduced Akt S-nitrosylation, while improving myocyte cross-sectional area and contractile protein expression [28]. Noticeably, Akt functions downstream of insulin-like growth factor-1 and stimulates glucose uptake, glycogen synthesis and protein synthesis. Akt also inhibits myonuclear apoptosis by interfering with mitochondrial outer membrane permeabilization. Indeed, $\mathrm{Wu}$ et al. [28] showed that amelioration of Akt activity following acetaminophen administration resulted in reduced levels of myonuclear apoptosis in the rat soleus muscle, sustained by decreased Bax expression and increased content of Bcl-2.

Further support to the usefulness of pharmacologically targeting myocyte apoptosis to rescue muscle mass arises from the observation that myostatin inhibition, obtained via administration of a mouse chimera of antihuman myostatin antibody, reduced the extent of myonuclear apoptosis in the tibialis anterior muscle of aged mice [29]. Furthermore, gene expression levels of caspase-3 were significantly lowered following antibody-directed myostatin inhibition. These biochemical changes were associated with increased myofiber cross-sectional area and enhanced maximum force generation of the tibialis anterior muscle [29]. Finally, Xu et al. [30] showed that administration of a nutraceutical mixture containing coenzyme Q10, creatine and ginseng extracts to late middle-aged rats decreased the susceptibility of quadriceps muscle subsarcolemmal mitochondria to undergo outer membrane permeabilization. The reduced apopto- tic potential translated into improvements in forelimb grip strength. Interestingly, these effects were not observed when the supplementation was initiated later in life, suggesting that a critical time window exists for this intervention to succeed.

With respect to the effects of hormonal interventions on age-related skeletal myocyte apoptosis, it has recently been reported that testosterone supplementation reduces the extent of myonuclear apoptosis in the gastrocnemius muscle of old mice, while improving muscle mass and fiber cross-sectional area [31]. These adaptations were likely mediated by the suppression of myostatin signaling induced by the hormonal intervention. In contrast to testosterone supplementation, administration of growth hormone $(\mathrm{GH})$ to old rats failed to reduce the severity of skeletal myocyte apoptosis and to improve muscle mass [25]. Indeed, GH supplementation, although reducing the expression of TNF-R 1 and active caspase- 8 in the extensor digitorum longus muscle, did not alleviate the agerelated elevation of cleaved caspase- 3 and apoptotic DNA fragmentation. Apoptosis was even exacerbated by GH in the soleus muscle. This was coupled with reduced grip strength in GH-supplemented rats compared with agematched controls.

Similar to GH supplementation, 2-week administration of human IL-15 to aged rats unexpectedly stimulated, rather than suppressed, myonuclear apoptosis [32]. As a result, IL-15-treated rats experienced a reduction in muscle weight relative to age-matched controls. The mechanisms underlying this paradoxical effect of IL-15 are not known. However, it may be hypothesized that heterologous IL-15 might have functioned as an IL-15 antagonist in rats. Alternatively, it is possible that the lack of a concomitant increase in IL-15R $\alpha$ expression, as observed with CR [8], might have provided IL-15 with unanticipated proapoptotic properties.

\section{Genetic Manipulations}

The most convincing evidence linking mitochondrial dysfunction, myonuclear apoptosis and sarcopenia comes from the characterization of mice expressing a proofreading deficient mitochondrial DNA (mtDNA) polymerase [33]. Due to a disruption in the exonuclease domain of the polymerase- $\gamma$, these rodents, called PolG mice, accumulate a high load of mtDNA mutations and display a premature aging phenotype, including severe sarcopenia. The loss of muscle mass in mtDNA mutator mice is accompanied by significant elevations in cleaved caspase -3 content, caspase- 3 and -9 activities, and DNA fragmentation, indicating that excessive myonuclear 
apoptosis drives the development of sarcopenia. It is noteworthy that 5 months of endurance exercise induced systemic mitochondrial biogenesis, prevented mtDNA mutations, improved mitochondrial function and morphology, and lowered levels of apoptosis in multiple tissues of PolG mice [34]. These adaptations conferred complete phenotypic protection, reduced multisystem pathology (including premature sarcopenia) and prevented early mortality.

Similar to the PolG model, mice lacking the antioxidant enzyme copper-zinc superoxide dismutase (Sod1 ${ }^{-/-}$) age prematurely and develop severe sarcopenia [35]. Histological analysis of muscle samples from Sod $1^{-/-}$mice showed smaller myofiber cross-sectional area and a selective loss of white/fast glycolytic fibers, two features also displayed by aged human muscles. Biochemical characterization of Sod $1^{-/-}$mice revealed an age-related increase in muscular mitochondrial reactive oxygen species generation coupled with mitochondrial functional decay. Interestingly, the susceptibility to permeability transition was higher in muscle mitochondria from Sod1 $1^{-/-}$rodents relative to wild-type controls, indicating an elevated apoptotic potential. Furthermore, levels of Bax and Bcl-2 homologous antagonist/killer (Bak) were significantly elevated in mitochondria from Sod1 $1^{-/}$mice, whereas Bcl-2 and $\mathrm{B}$-cell lymphoma-extra large $\left(\mathrm{Bcl}-\mathrm{X}_{\mathrm{L}}\right)$ were downregulated. These alterations translated into increases in caspase- 3 activity and apoptotic DNA fragmentation. Moreover, ultrastructural analysis of gastrocnemius muscle sections showed that apoptotic myonuclei were surrounded by abnormal mitochondria, indicating that oxidative stress-induced mitochondrial dysfunction instigates apoptosis. This observation is of great interest since it suggests that during the development of sarcopenia, apoptosis may occur independently of nuclear dysfunction. In this scenario, myonuclear apoptosis might therefore represent a fatality, whose prevention, via selective antiapoptotic interventions, may rescue myofibers from atrophy. This hypothesis is further supported by the observation that muscle-specific overexpression of peroxisome proliferator-activated receptor- $\gamma$ coactivator- $\alpha$ (PGC- $1 \alpha$ ), a master regulator of mitochondrial biogenesis, preserves mitochondrial function, reduces oxidative damage and decreases the activation of myonuclear apoptosis in aged mice [36]. Indeed, expression levels of $\mathrm{Bcl}-2$ were increased and those of Bax decreased in muscles from old PGC- $1 \alpha$ transgenic mice compared with age-matched wild-type rodents. Furthermore, protein expression of cleaved caspase- 3 as well as the extent of apoptotic DNA fragmentation were significantly blunted in aged muscles overex- pressing PGC- $1 \alpha$. As a result, old transgenic rodents exhibited greater muscle mass and physical performance compared with age-matched wild-type mice.

\section{Conclusions}

Available evidence supports the hypothesis that excessive myonuclear apoptotic elimination may drive the onset and progression of sarcopenia. Furthermore, results from studies employing behavioral, pharmacological or genetic interventions to rescue muscle mass at old age indicate that preservation of muscle integrity is accompanied, and perhaps mediated, by decreases in the extent of myocyte apoptosis (table 1). These findings raise the possibility that specifically targeting myonuclear apoptosis might provide a novel and effective therapeutic tool to counter sarcopenia. Of course, other cellular processes exist that contribute to muscle aging, including altered protein turnover, impaired satellite cell function and dysfunctional autophagy. Therefore, it is possible that simply downregulating myonuclear apoptosis might not completely preserve muscle mass and function into advanced age. In this scenario, combination therapies also targeting muscle protein turnover, autophagy and satellite cell function may provide a more thorough protection against muscle aging. Furthermore, devising an effective muscle-specific antiapoptotic intervention will face several challenges, including the identification of the most relevant apoptotic pathway(s) to target, the time window within which the intervention needs to be applied and, of course, safety issues. However, the possibility of relieving the individual and societal burden associated with sarcopenia and physical frailty at old age makes this task worth pursuing.

References

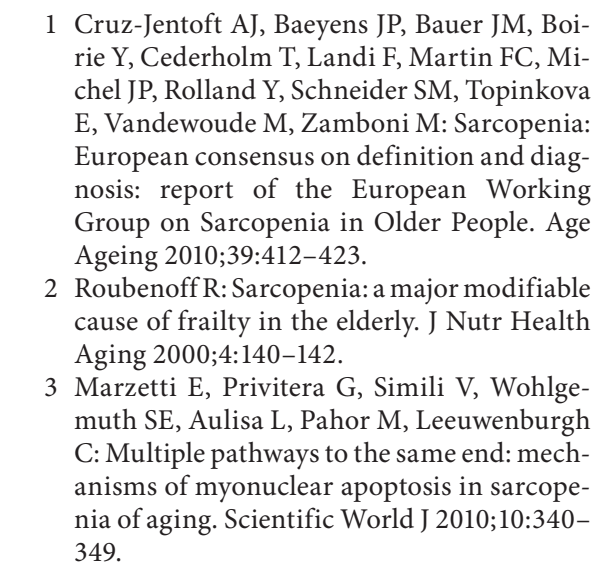

Gerontology 2012;58:99-106 
-4 Marzetti E, Hwang JC, Lees HA, Wohlgemuth SE, Dupont-Versteegden EE, Carter CS, Bernabei R, Leeuwenburgh C: Mitochondrial death effectors: relevance to sarcopenia and disuse muscle atrophy. Biochim Biophys Acta 2010;1800:235-244.

5 Walston J, Fedarko N, Yang H, Leng S, Beamer B, Espinoza S, Lipton A, Zheng H, Becker $\mathrm{K}$ : The physical and biological characterization of a frail mouse model. J Gerontol A Biol Sci Med Sci 2008;63:391-398.

-6 Marzetti E, Wohlgemuth SE, Lees HA, Chung HY, Giovannini S, Leeuwenburgh C: Age-related activation of mitochondrial caspase-independent apoptotic signaling in rat gastrocnemius muscle. Mech Ageing Dev 2008; 129:542-549.

7 Phillips T, Leeuwenburgh C: Muscle fiber specific apoptosis and TNF-alpha signaling in sarcopenia are attenuated by life-long calorie restriction. FASEB J 2005;19:668-670.

-8 Marzetti E, Carter CS, Wohlgemuth SE, Lees HA, Giovannini S, Anderson B, Quinn LS, Leeuwenburgh C: Changes in IL-15 expression and death-receptor apoptotic signaling in rat gastrocnemius muscle with aging and life-long calorie restriction. Mech Ageing Dev 2009;130:272-280.

-9 Turpin SM, Lancaster GI, Darby I, Febbraio MA, Watt MJ: Apoptosis in skeletal muscle myotubes is induced by ceramides and is positively related to insulin resistance. Am J Physiol Endocrinol Metab 2006;291:E1341E1350.

10 Kerr JF, Wyllie AH, Currie AR: Apoptosis: a basic biological phenomenon with wideranging implications in tissue kinetics. $\mathrm{Br} \mathrm{J}$ Cancer 1972;26:239-257.

- 11 Zimmermann KC, Bonzon C, Green DR: The machinery of programmed cell death. Pharmacol Ther 2001;92:57-70.

12 Parsons MJ, Green DR: Mitochondria in cell death. Essays Biochem 2010;47:99-114.

-13 Du J, Wang X, Miereles C, Bailey JL, Debigare R, Zheng B, Price SR, Mitch WE: Activation of caspase- 3 is an initial step triggering accelerated muscle proteolysis in catabolic conditions. J Clin Invest 2004;113:115-123.

14 Argiles JM, Lopez-Soriano FJ, Busquets S: Apoptosis signalling is essential and precedes protein degradation in wasting skeletal muscle during catabolic conditions. Int J Biochem Cell Biol 2008;40:1674-1678.

-15 Marzetti E, Lees HA, Wohlgemuth SE, Leeuwenburgh C: Sarcopenia of aging: underlying cellular mechanisms and protection by calorie restriction. Bio Factors 2009;35:28-35.

16 Dirks AJ, Leeuwenburgh C: Aging and lifelong calorie restriction result in adaptations of skeletal muscle apoptosis repressor, apoptosis-inducing factor, X-linked inhibitor of apoptosis, caspase-3, and caspase-12. Free Radic Biol Med 2004;36:27-39.
17 Ekhterae D, Lin Z, Lundberg MS, Crow MT, Brosius FC, III, Nunez G: ARC inhibits cytochrome $\mathrm{c}$ release from mitochondria and protects against hypoxia-induced apoptosis in heart-derived H9c2 cells. Circ Res 1999; 85:e70-e77.

18 Landi F, Zuccala G, Gambassi G, Incalzi RA, Manigrasso L, Pagano F, Carbonin P, Bernabei R: Body mass index and mortality among older people living in the community. J Am Geriatr Soc 1999;47:1072-1076.

19 Chung S, Yao H, Caito S, Hwang JW, Arunachalam G, Rahman I: Regulation of SIRT1 in cellular functions: role of polyphenols. Arch Biochem Biophys 2010;501:79 90.

-20 Siddiqui MA, Kashyap MP, Kumar V, AlKhedhairy AA, Musarrat J, Pant AB: Protective potential of trans-resveratrol against 4-hydroxynonenal induced damage in $\mathrm{PC} 12$ cells. Toxicol In Vitro 2010;24:1592-1598.

21 Jackson JR, Ryan MJ, Hao Y, Alway SE: Mediation of endogenous antioxidant enzymes and apoptotic signaling by resveratrol following muscle disuse in the gastrocnemius muscles of young and old rats. Am J Physiol Regul Integr Comp Physiol 2010;299:R1572R1581.

22 Frankel JE, Bean JF, Frontera WR: Exercise in the elderly: research and clinical practice. Clin Geriatr Med 2006;22:239-256.

23 Fiatarone MA, O'Neill EF, Ryan ND, Clements KM, Solares GR, Nelson ME, Roberts SB, Kehayias JJ, Lipsitz LA, Evans WJ: Exercise training and nutritional supplementation for physical frailty in very elderly people. N Engl J Med 1994;330:1769-1775.

24 Song W, Kwak HB, Lawler JM: Exercise training attenuates age-induced changes in apoptotic signaling in rat skeletal muscle. Antioxid Redox Signal 2006;8:517-528.

25 Marzetti E, Groban L, Wohlgemuth SE, Lees HA, Lin M, Jobe H, Giovannini S, Leeuwenburgh C, Carter CS: Effects of short-term GH supplementation and treadmill exercise training on physical performance and skeletal muscle apoptosis in old rats. Am J Physiol Regul Integr Comp Physiol 2008;294: R558-R567.

26 Narkar VA, Downes M, Yu RT, Embler E, Wang YX, Banayo E, Mihaylova MM, Nelson MC, Zou Y, Juguilon H, Kang H, Shaw RJ, Evans RM: AMPK and PPARdelta agonists are exercise mimetics. Cell 2008;134:405415.
27 Carter CS, Giovaninni S, Seo DO, Dupree J, Morgan D, Chung HY, Lees H, Daniels M, Hubbard GB, Lee S, Ikeno Y, Foster TC, Buford TW, Marzetti E: Differential effects of enalapril and losartan on body composition and indices of muscle quality in aged male Fischer $344 \times$ Brown Norway rats. Age (Dordr) 2011;33:167-183.

28 Wu M, Katta A, Gadde MK, Liu H, Kakarla SK, Fannin J, Paturi S, Arvapalli RK, Rice KM, Wang Y, Blough ER: Aging-associated dysfunction of Akt/protein kinase B: S-nitrosylation and acetaminophen intervention. PLoS One 2009;4:e6430.

-29 Murphy KT, Koopman R, Naim T, Leger B, Trieu J, Ibebunjo C, Lynch GS: Antibody-directed myostatin inhibition in 21-mo-old mice reveals novel roles for myostatin signaling in skeletal muscle structure and function. FASEB J 2010;24:4433-4442.

- $30 \mathrm{Xu}$ J, Seo AY, Vorobyeva DA, Carter CS, Anton SD, Lezza AM, Leeuwenburgh C: Beneficial effects of a Q-ter based nutritional mixture on functional performance, mitochondrial function, and oxidative stress in rats. PLoS One 2010;5:e10572.

- 31 Kovacheva EL, Hikim AP, Shen R, Sinha I, Sinha-Hikim I: Testosterone supplementation reverses sarcopenia in aging through regulation of myostatin, c-Jun NH2-terminal kinase, Notch, and Akt signaling pathways. Endocrinology 2010;151:628-638.

32 Pistilli EE, Alway SE: Systemic elevation of interleukin-15 in vivo promotes apoptosis in skeletal muscles of young adult and aged rats. Biochem Biophys Res Commun 2008;373: 20-24.

33 Kujoth GC, Leeuwenburgh C, Prolla TA: Mitochondrial DNA mutations and apoptosis in mammalian aging. Cancer Res 2006;66: 7386-7389.

-34 Safdar A, Bourgeois JM, Ogborn DI, Little JP, Hettinga BP, Akhtar M, Thompson JE, Melov S, Mocellin NJ, Kujoth GC, Prolla TA, Tarnopolsky MA: Endurance exercise rescues progeroid aging and induces systemic mitochondrial rejuvenation in mtDNA mutator mice. Proc Natl Acad Sci USA 2011;108: 4135-4140.

35 Jang YC, Lustgarten MS, Liu Y, Muller FL, Bhattacharya A, Liang $\mathrm{H}$, Salmon $\mathrm{AB}$, Brooks SV, Larkin L, Hayworth CR, Richardson A, Van RH: Increased superoxide in vivo accelerates age-associated muscle atrophy through mitochondrial dysfunction and neuromuscular junction degeneration. FASEB J 2010;24:1376-1390.

-36 Wenz T, Rossi SG, Rotundo RL, Spiegelman $\mathrm{BM}$, Moraes CT: Increased muscle PGC-1alpha expression protects from sarcopenia and metabolic disease during aging. Proc Natl Acad Sci USA 2009;106:20405-20410. 